\title{
OUTCOMES OF EPILEPSY SURGERY FOR MEDICALLY REFRACTORY TEMPORAL LOBE EPILEPSY IN OLDER PATIENTS
}

Authors: Temenuzhka Mihaylova, Fawad Khan, Henry A. Buchtel, Oren Sagher, Linda M. Selwa Authors and Institutional Affiliations: Temenuzhka Mihaylova ${ }^{1}$, Fawad Khan ${ }^{4}$, Henry A. Buchtel $^{3}$, Oren Sagher ${ }^{2}$, Linda M. Selwa ${ }^{1}$

${ }^{1}$ Department of Neurology, University of Michigan, Ann Arbor, Mi

${ }^{2}$ Department of Neurosurgery, University of Michigan, Ann Arbor, Mi

${ }^{3}$ Department of VA Ann Arbor and University of Michigan, Departments of Psychiatry and Psychology, Ann Arbor, Mi

${ }^{4}$ The International Center for Epilepsy at Ochsner, Ochsner Neurosciences Institute, New Orleans, LA

Corresponding author:

Temenuzhka Mihaylova, MD, PhD

University of Michigan

Department of Neurology

1500 East Medical Center Dr.

Ann Arbor, MI 48109

Phone: \#734-936-7310

Fax: \#734-936-5520

Email: tmihaylo@med.umich.edu

Email addresses of each co-author:

Fawad Khan - khanv6@yahoo.com

Henry A. Buchtel - gusb@med.umich.edu

Oren Sagher - osagher@med.umich.edu

Linda Selwa - lmselwa@med.umich.edu

\begin{abstract}
Purpose-Resective epilepsy surgery for temporal lobe epilepsy (TLE) remains underutilized in elderly patients and only few studies report postsurgical seizure and neurocognitive outcomes in this group. The aim of this study was to investigate the tolerability and efficacy of surgery in older epileptics.

Methods-We conducted a retrospective analysis of 18 patients aged 55 or older to assess the efficacy and tolerability of the procedure. Mean disease duration was 34 years.

Results-Fourteen patients (78\%) achieved Engel class I outcome over a mean follow-up period of 4.2 years. Thirteen patients had neuroimaging and histopathology consistent with mesial temporal sclerosis (MTS). We did not find a measurable effect of disease duration on postsurgical outcome. We confirmed
\end{abstract}


that the MRI evidence of MTS can be used as a predictor for postsurgical outcome. Postsurgical neuropsychological evaluation completed in 13 patients between three months and two and a half years post resection showed decline in verbal abilities most significantly in those who had undergone dominant temporal lobe surgery.

Discussion/Conclusion-Our results indicate that epilepsy surgery is well tolerated, safe and effective in older patients with refractory TLE. Postsurgical neuropsychological evaluation showed expected decline in verbal abilities in patients who had undergone dominant resection.

Keywords-temporal lobe epilepsy; elderly patients; surgical outcome; cognitive function; brain MRI; seizure frequency 


\section{INTRODUCTION}

Resective epilepsy surgery for medically refractory temporal lobe epilepsy (MRTLE) has been increasingly well-supported as an effective treatment for patients with pharmacoresistant focal epilepsy but remains underutilized in the elderly and its safety and efficacy in that population is rarely reported. The incidence of epilepsy in the general population is reported in the 30$80 / 100,000$ range and the prevalence is 510/1,000. A recent epidemiologic study found a prevalence among seniors of $10.8 / 1,000$ and an estimated yearly incidence of new-onset epilepsy of 2.4/1,000 [8]. There is skepticism about outcomes in older patients treated with surgery related to the presence of multiple comorbidities, preexisting cognitive decline and longer seizure duration $[2,13]$. Few studies report postsurgical outcomes in older adults and the available data are based on small number heterogeneous samples. However, few articles have reported encouraging results comparable to those in younger patients with refractory TLE $[4,5,20]$. There are only a few studies in older adults which evaluate postsurgical neurocognitive outcome.

\section{Material and Methods}

The goal of this retrospective cohort study is to assess the efficacy and tolerability of surgery in patients older than 55 years with history of MRTLE. We reviewed a computer database for all patients with TLE $(\mathrm{N}=381)$ who underwent epilepsy surgery from 1999 to 2011 at our institution. Eighteen patents met the inclusion criteria for age at surgery 55 or older and history of MRTLE. One patient had undergone two surgeries at age of 55 and 57, respectively. Patients with both lesional and nonlesional imaging characteristics were included and were surgically treated by the same neurosurgeon (OS) at the University of Michigan.

To determine outcomes, we looked at different parameters such as age at seizure onset, seizure semiology, frequency and duration, number of failed medications, results of standard presurgical workup, histopathology and postsurgical seizure reduction. Neuropsychological evaluation as part of the standard presurgical workup was completed in all patients and postoperative testing was performed in 13 patients between 3 months and $21 / 2$ years after resection (7 left and 6 right temporal lobe resections). We compared presurgical and postsurgical test results in order to assess surgery impact on cognitive functioning. For the purposes of the study we analyzed verbal and visual memory scores in addition to full scale IQ (FSIQ), verbal IQ (VIQ), and performance IQ (PIQ). The following tests were utilized: Wechsler Adult Intelligence Scale-Revised, Wechsler Memory Scale (specific subscales when needed for reevaluation), Phonemic Verbal Fluency and Boston Naming Test, Mini-Mental State Exam, Finger Oscillation and Grip Strength, Sensory-Perceptual Screen (Visual and Tactile), Trail Making Test (A\&B), Clinical Interview, Self-Rating Scale, Objective Personality Assessment (MMPI-2 or MiniMulti Personality Screen), Neuropsychological Patient History (Time 1) and Patient History Update (Time 2). Presurgical data included results from longterm video EEG monitoring (LTM), brain MRI with epilepsy protocol, neuropsychological assessment, speech and language evaluation and WADA test. Selected patients whose clinicoelectrophysiological data were discordant also underwent SPECT or/and PET scan. Intracranial EEG recording was performed in small number of patients whose noninvasive recordings were inconclusive. The type of surgical resection, 
anterior temporal lobectomy (ATL), $4-5 \mathrm{~cm}$ or $3-4 \mathrm{~cm}$ from the temporal tip in nondominant and dominant hemisphere, respectively versus selective amygdalohippocampectomy (AH) was chosen individually based upon presurgical workup results. The degree of postsurgical seizure reduction was reported using the modified Engel classification according to which class I outcome was categorized as seizure freedom, class II - rare (1-3 seizures/year ) or only nocturnal seizures, class III - seizure reduction greater than 90\% and class IV - improvement less than $90 \%$.

\section{Results}

Eighteen patients aged 55 or older underwent epilepsy surgery for medically refractory temporal lobe seizures. Eleven were men and seven were women. The age at surgery ranged from 55 to 66 years with mean age of 58 . The age at seizure onset was from 6 months to 57 years with mean age of 21.3 years. Epilepsy duration was identified as the interval between the date of the first afebrile seizure and the date of the surgery. Mean epilepsy duration in our cohort was 34 years, ranging from 3 years to 65 years.

All patients had localization-related epilepsy with focal seizures with dyscognitive features (complex partial seizures); two patients had focal seizures with retained awareness (simple partial seizures). Ten had secondarily generalized convulsive seizures. Five patients had reported history suggestive of structural-metabolic (symptomatic) etiology (four had CNS infection and one had head trauma), and 13 patients had unknown etiology. Seizure frequency varied from 7 seizures per week to 1 seizure per month. The mean number of failed anticonvulsants was 6.6 (ranged from 1 to $10)$.

Prolonged scalp video-EEG recordings demonstrated left temporal onset in 11 patients $(61 \%)$ and right temporal onset in 7 patients (one patient had left and right temporal electrographic onset but further pre-surgical workup localized the ictus to the right temporal lobe). The interictal and ictal EEG findings were concordant in all patients.

Intracranial recording (intraoperative or extraoperative) was performed in five patients for seizure localization or language lateralization purposes.

Pre-surgical brain MRI findings are delineated in Table 1. Fourteen patients (78\%) had MRI evidence of mesial temporal sclerosis (MTS), three had other lesions, and one patient had a normal MRI.

Table 1. MRI abnormalities

\begin{tabular}{|l|l|}
\hline MTS (n) & Other Lesion or no lesion (n) \\
\hline L MTS(+/- atrophy) ( 8) & Subcortical R middle temporal gyrus cystic lesion (1) \\
\hline R MTS(+/- atrophy) (3) & L mesial temporal cavernous malformation (1) \\
\hline
\end{tabular}




\begin{tabular}{|l|l|}
\hline bilateral MTS (R>L) (3) & L mesial temporal cystic encephalomalacia (1) \\
\hline & Normal (1) \\
\hline
\end{tabular}

MTS - mesial temporal sclerosis, R - right, L - left

Seven patients needed additional functional neuroimaging with either ictal SPECT $(n=5)$ or PET scan $(\mathrm{N}=2)$ and two of those patients had both studies. The test results were concordant with the other indices of lateralization and localization.

The surgical approach included ATL in 8 patients and selective $\mathrm{AH}$ in 10. Selective resection was preferred intervention in most of the patients with dominant lobe epilepsy.

Resected tissue was examined by a neuropathologist and the data on each patient is based on the official neuropathology report. The patients with MRI evidence of MTS showed a variable degree of CA-1 neuronal loss and gliosis. One of these patients also had dual pathology - histologic evidence of multifocal cortical dysplasia. Two of the patients with "other lesion" on MRI had gangliogliomas and one had a cavernous malformation. The patient with normal MRI had undergone selective left $\mathrm{AH}$ and his histopathology was consistent with neuronal loss and gliosis.

During mean follow-up of 4.2 years (ranged from 1 year to 12 years) Engel Class I outcome was achieved in 14 patients $(78 \%)$ of whom 13 patients had neuroimaging and histopathology evidence consistent with MTS and one patient had a normal neuroimaging but similar histopathology. The remaining 4 patients ranged from Engel class II to IV. The patient with dual pathology and the three patients with bilateral MTS all had Engel Class I outcome. Nine patients underwent $\mathrm{AH}$ and nine patients ATL. There was no difference in the postsurgical outcomes in the subsets, seven patients in each group had Engel Class I outcome. The rate of seizure control remained steady over the individual followup periods.

All patients had presurgical neuropsychometric evaluation. The results were classified into three categories: below average (Quotient <90), average (Quotient 90-109) and above average (Quotient 110+). On verbal memory testing, $15(83.3 \%)$ patients tested below average and $3(16.7 \%)$ patients were rated average. On visual memory testing, $12(66.7 \%)$ patients tested below average, $4(22.2 \%)$ patients tested at average and $2(11.1 \%)$ patients were above average. Overall, total of 15 patients $(83.3 \%)$ were below average at baseline. (Table2)

Table 2. Presurgical memory scores. $n=18$

\begin{tabular}{|l|l|l|l|}
\hline Memory scores & Below average n & Average n & Above average n \\
\hline Verbal memory & $15 *(83.3 \%)$ & $3 *(16.7 \%)$ & 0 \\
\hline
\end{tabular}




\begin{tabular}{|l|l|l|l|}
\hline Visual memory & $12 *(66.7 \%)$ & $4 *(22.2 \%)$ & $2(11.1 \%)$ \\
\hline
\end{tabular}

*Patients exceed $\mathrm{n}=18$ due to patient overlap

Thirteen patients had postsurgical neuropsychometric testing (five patients did not come in for their appointments). The results were classified as declined, stable and improved. On verbal memory testing, 5
(38.5\%) patients showed decline, $6(46.1 \%)$ were stable and $2(15.4 \%)$ improved. On visual memory testing, $4(30.8 \%)$ patients showed decline, 7 (53.8\%) were stable and 2 (15.4\%) improved. (Table 3)

Table 3. Postsurgical memory scores. $n=13$

\begin{tabular}{|l|l|l|l|}
\hline Memory scores & Declined ( n ) & Stable ( n ) & Improved ( n ) \\
\hline Verbal memory & $5 *(38.5 \%)$ & $6 *(46.1 \%)$ & $2(15.4 \%)$ \\
\hline Visual memory & $4 *(30.8 \%)$ & $7 *(53.8 \%)$ & $2(15.4 \%)$ \\
\hline
\end{tabular}

* Patients exceed $n=13$ due to patient overlap

Total of five patients (38.5\%) showed decline in both verbal and visual memory functions. Four of them had Engel Class I seizure outcome and one had Class III. Four patients had left sided resection.

Neuroimaging showed evidence of left MTS $(\mathrm{n}=2)$, right MTS $(\mathrm{n}=1)$, left mesial temporal cavernoma $(n=1)$ and normal MRI $(n=1)$.
All patients with improved either verbal or visual memory scores $(n=4)$ had right-sided resection and variable seizure control: two had Class I, one had Class II and one had Class IV. Neuroimaging showed right MTS $(\mathrm{n}=3)$ and right middle temporal cystic lesion $(n=1)$.

Figure 1 represents the actual verbal and visual memory score distribution pre- and post-surgery. 
Figure 1. Actual Memory scores pre- and post-surgery

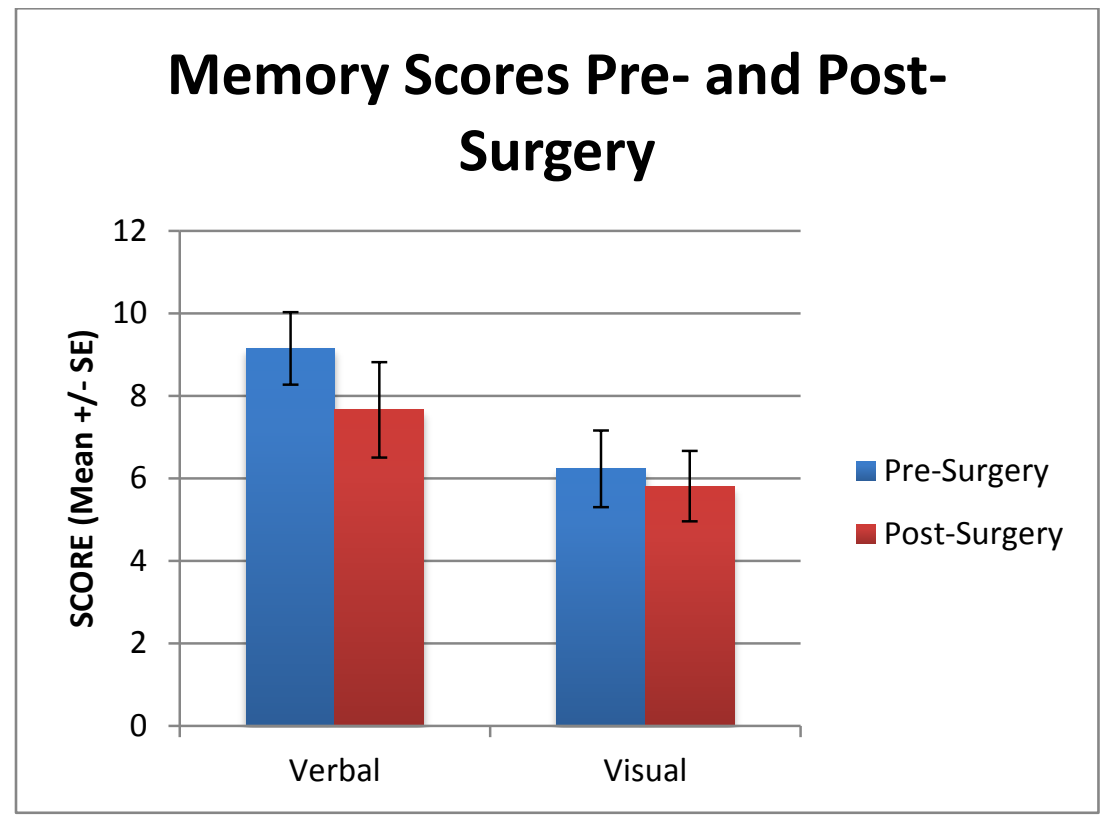

Mean presurgical FSIQ was found at 91.8 and mean postsurgical FSIQ was 88.3. Preoperative and postoperative mean VIQ was 93.4 and 89.7, respectively. Mean PIQ remained overall stable, 90.5 preoperatively and 90.8 postoperatively (Figure 2 ). 
Figure 2. Mean IQ scores

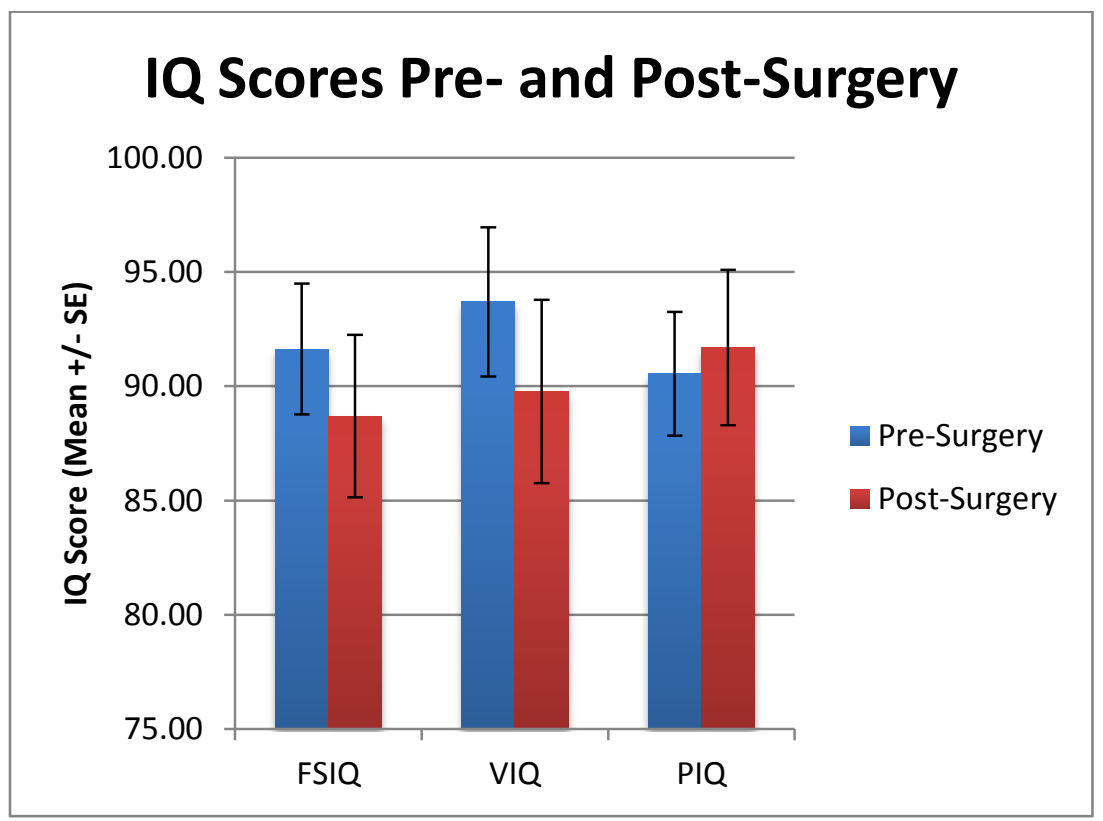

FSIQ-full scale IQ, VIQ-verbal IQ, PIQperformance IQ

respectively); these patients also had a tendency for decreased visual memory $(p<0.057)$ without statistical significance.

Five patients did not have a formal postoperative cognitive testing. Of note, no clinical evidence of deteriorated cognitive status was found upon detailed review of patients' follow-up notes.

A statistical analysis of neuropsychological scores shows that in patients with left resection verbal IQ and verbal memory declined after surgery $(p<0.024$ and $p<0.047$

In patients with right resection, there was a tendency for visual memory decline $(\mathrm{p}<0.058)$ and for Full Scale IQ decline $(p<0.055)$ after surgery but none of these findings reached statistical significance and none of the other abilities were significantly affected, including Verbal IQ, Performance IQ and verbal memory (Table 4).

Table 4. t-test results

\begin{tabular}{|l|r|l|l|}
\hline t-tests & L-surgery $(\mathrm{n}=7)$ & \multicolumn{2}{l|}{ R-surgery $(\mathrm{n}=6)$} \\
\hline & \multicolumn{2}{|l|}{ p value } & p value \\
\hline pre-post FSIQ & 0.149 & & 0.055 \\
\hline pre-post VIQ & $\mathbf{0 . 0 3 8}$ & & 0.226 \\
\hline pre-post PIQ & 0.436 & & 0.199 \\
\hline
\end{tabular}




\begin{tabular}{|l|r|r|r|}
\hline pre-post Verbal memory & $\mathbf{0 . 0 4 7}$ & & 0.483 \\
\hline pre-post Visual memory & 0.057 & & 0.058 \\
\hline
\end{tabular}

FSIQ-full scale IQ, VIQ-verbal IQ, PIQ-performance IQ

In order to determine independent predictors of outcome, we performed bivariate analysis (Chi-square, ANOVA, t-test) for gender, type of surgery, duration of epilepsy, type of MRI lesion, Engel class outcome and cognitive outcome. Statistical analysis found no significant difference when outcome class was correlated to epilepsy duration, number of failed medications, neuroimaging findings, and type of epilepsy surgery.

\section{Discussion}

Epilepsy is a common condition in older patients with significant consequences for quality of life. As the age profile of the general population changes, there will likely continue to be a significant increase in the number of older adults in the population. It is estimated that the number of people aged 65 and older will double between 2010 and 2050. A fourfold increase is expected among persons over the age of 85 [18].

This mandates a careful study of the disease in older population, its management approach and impact on different aspects of life. It is important to identify predictors of cognitive outcomes in addition to seizure outcome.

Our results indicate that the epilepsy surgery is well tolerated, safe and efficacious in patients aged 55 and above who have a longstanding history (mean duration 34 years) of refractory temporal lobe epilepsy that is in general agreement with previous reports in older patients [1, 5]. Seizure freedom was achieved in $78 \%$ of the patients and the results remained steady over 4.2 year mean follow-up period.

Our results confirmed that there were no significant differences in surgical outcome between those patients having a larger resection and those patients having a selective hippocampal removal [12].

Boling et al. [4] conducted a review of 18 patients 50 years and older with lesional (nontumoral) TLE treated surgically and mean disease duration of 34.9 years who were compared to younger individuals. $83 \%$ obtained meaningful improvement, becoming either seizure free or experiencing only rare seizure.

Long seizure duration did not reduce the ability of surgery to produce seizure freedom similar to our results.

Srikijvilaiku et al. [21] reported statistically significant seizure-free outcome in $56.2 \%$ of 16 patients over 50 years with history of refractory epilepsy and MTS, mean disease duration of 30.8 years and mean follow-up period of 2.85 years. This was compared to a younger group of 184 patients with the same clinical condition aged below 50 years of whom $79.3 \%$ achieved seizure freedom.

Grivas et al. [9] studied 52 patients older than 50 years operated on for intractable mesial or combined mesiolateral TLE and found that $71 \%$ had complete seizure control and $19 \%$ had only rare postoperative seizures. 
A recent study [15] in 21 patients, 50 or older, all with MTS and long follow-up period of 9.57 years found compatible seizure free rate in older ( $81 \%$ class I) and young patients ( $83 \%$ class I). The authors did not find a measurable effect of disease duration on postsurgical outcome and showed lower complication rate in the older group. Similar results were reported by Costello et al. [6].

However, there are conflicting data in the literature on whether long seizure duration affects outcome. Sirven et al. [20] compared postsurgical seizure outcome, neuropsychological test scoring and driving status in 30 patients aged 50 and older (median age 51.9) to 340 younger patients (median age 32.8). $53 \%$ of the older patients were seizure free as compared to $75 \%$ in the younger group. The authors found that shorter duration of epilepsy and younger age at surgery was associated with seizure relief, findings that were not confirmed in our study.

Negative correlation between increasing age at surgery and likelihood of favorable outcome following temporal lobectomy were reported by several groups $[3,10,11]$. In our study the longer disease duration does not appear to play a major role in postsurgical outcomes.

The type of the MRI lesion can be used as a predictor for postsurgical outcome and our results confirm the widely accepted idea of higher seizure freedom expected in patients with lesional study, specifically MTS and concordant presurgical data.

We looked at baseline (presurgical) cognitive performance and compared to postoperative functioning across several cognitive domains: verbal memory, visual memory, FSIQ, VIQ and PIQ. At baseline, the majority of our patients $(83.3 \%)$ had below average verbal and visual scores (table 2). Postoperatively, five patients (38.5\%) showed decline in both verbal and visual memory despite excellent seizure control in four of them (table 3). All but one of these patients had left-sided resections. Several patients $(n=4)$ had improved visual or verbal memory but poorer seizure control; all of them had undergone right-sided resection. Statistically significant decline in verbal IQ and verbal memory was found in patients who underwent left surgery (table 4). Patients with right resection had a tendency for visual memory and FSIQ decline but the results did not reach statistical significance. Similar findings had been published previously [16, 17, 19]. Our findings are consistent with already reported verbal memory deterioration following dominant temporal lobe surgery $[7,18,19$, 20].

Overall, we did not find strong evidence of increased vulnerability of cognitive functions in older patients as compared to other reported TLE groups. Murphy et al. [15] found no evidence of different neuropsychological outcomes for older than 50 patients as opposed to younger patients. Cascino et al. [5] and McLachlan et al. [14] reported little to no postoperative change in IQ and memory in their older groups.

Statistical relationships between outcome and epilepsy duration, number of failed medications, MRI findings and type of surgery were not found in our study but were limited by small sample size.

Limitations of the study include retrospective analysis of a small sample size that yielded low statistical power and thus potentially limited the ability to detect significant results, although such small sample can be expected in this patient population that is rarely referred for surgical treatment. Another major limitation is that our study did not have a younger control 
group. The impact of biological risk factors such as history of cerebrovascular disease, neurodegenerative disease, cardiovascular disease, etc. has not been assessed. Our results contribute to the understanding of whether older patients undergoing surgery are at increased risk for cognitive decline. We found little evidence of any problems specific to the aging. Comparison to postoperative neuropsychometric outcomes in a younger group is needed to confirm the hypothesis that later age at seizure onset is associated with greater risk for memory decline following temporal lobe resection. Longer postsurgical follow-up period and larger study group may be helpful in the future. Our results are only applicable to patients with TLE, predominantly mesial temporal epilepsy, but are not representative for older patients with other types of focal epilepsy. Our follow-up period of 4.2 years is substantially long but we recognize it would be advantageous to have even longer follow-up to assess the impact of surgery on later life degenerative disorders specifically in patients who undergo mesial temporal resection.

We have not reviewed factors determining quality of life such as driving status, independence of living, medication withdrawal, postsurgical complications, etc.

\section{CONCLUSION}

The aim of this retrospective study was to demonstrate that the epilepsy surgery in older patients with medically refractory TLE is well tolerated, safe and efficacious treatment approach. Long disease duration did not reduce the ability of surgery to produce seizure freedom. The presence of MTS in association with concordant postsurgical data can be used as a predictor for favorable postsurgical outcome as in younger patient populations. Overall, we did not find strong evidence of increased vulnerability of cognitive functions in older patients. Verbal memory deterioration is expected following dominant temporal lobe surgery similar to the results in younger cohorts. We hope that our findings are adding important insights concerning the management of refractory epilepsy in older epileptics and will reduce the skepticism about postsurgical outcomes in this patient group.

\section{CONFLICT OF INTEREST}

None of the authors has any conflict of interest to disclose.

\section{ACKNOWLEDGEMENTS}

We are grateful to all patients who participated in the study. 


\section{REFERENCES}

[1] Acosta I., F. Vale, W.O. $4^{\text {th }}$ Tatum, and S.R. Benbadis. 2008. "Epilepsy surgery after age 60.” Epilepsy Behav 12 (2):324-5.

[2] Bengzon A.R., T. Rasmussen, P. Gloor, J. Dussault, and M. Stephens.1968.

"Prognostic factors in the surgical treatment of temporal lobe epileptics." Neurology 18(18):717-31.

[3] Blume W.T., H. B. Desai, J. P. Girvin, R.S. McLachlan, and J.F. Lemieux.1994. "Effectiveness of temporal lobectomy measured by yearly follow-up and multivariate analysis." J Epilepsy 7(3):20314.

[4] Boling W., F. Andermann, D. Reutens, F. Dubeau, L. Caporicci, and A. Olivier.2001. "Surgery for temporal lobe epilepsy in older patients." J Neurosurg 95(2):242-8.

[5] Cascino G.D., F. W. Sharbrough, K. A. Hirschorn, and W.R. Marsh. 1991. "Surgery for focal epilepsy in the older patient." Neurology 41(9) :1415-1417.

[6] Costello D.J., D.C. Shields, S.S Cash, E.N. Eskandar, G.R. Cosgrove, and A.J. Cole. 2009."Consideration of epilepsy surgery in adults should be independent of age." Clin Neurol Neurosurg 111(3):240-5. doi: 10.1016/j.clineuro.2008.10.005.

[7] Dulay M.F. and R. M. Busch. 2012. "Prediction of neuropsychological outcome after resection of temporal and extratemporal seizure foci." Neurosurg Focus 32(3), E4.

doi:10.3171/2012.1.FOCUS11340.

[8] Faught E., J. Richman, R. Martin, E. Funkhouser, R. Foushee, P. Kratt, Y. Kim, K. Clements, N. Cohen, D. Adoboe, R. Knowlton, and M. Pisu. 2012. "Incidence and prevalence of epilepsy among older US
Medicare beneficiaries.” Neurology 78 (7):

448-53. doi: 10.1212/WNL.0b013e3182477edc. Epub 2012 Jan 18.

[9] Grivas A., J. Schramm, T. Kral, M. von Lehe, C. Helmstaedter, C.E. Elger, and H. Clusmann. 2006. "Surgical Treatment for Refractory Temporal Lobe Epilepsy in the Elderly: Seizure Outcome and Neuropsychological Sequels Compared with a Younger Cohort.” Epilepsia 47 (8):36472.

[10] Janszky J., I. Janszky, R. Schulz, M. Hoppe, F. Behne, H.W. Pannek, and A. Ebner. 2005. "Temporal lobe epilepsy with hippocampal sclerosis: predictors for longterm surgical outcome." Brain 128(Pt 2):395-404.

[11] Jeong S.W., S. K. Lee, K.K. Kim, H. Kim, J.Y. Kim, and C.K. Chung. 1999. "Prognostic factors in anterior temporal lobe resections for mesial temporal lobe epilepsy: multivariate analysis." Epilepsia 40(12):1735-9.

[12]Leonard G. 1991. "Temporal lobe surgery for epilepsy: neuropsychological variables related to surgical outcome." Can J Neurol Sci 18(4 Suppl):593-7.

[13] Malmgren K., M. Sullivan, G. Ekstedt, G. Kullberg, and E. Kumlien.1997. "Healthrelated quality of life after epilepsy surgery: a Swedish multicenter study." Epilepsia 38(7): 830-8.

[14] McLachlan R.S., C. J. Chovaz, W. T. Blume, and J.P. Girvin. 1992. "Temporal lobectomy for intractable epilepsy in patients over age 45 years." Neurology 42 (3 Pt 1):662-5.

[15] Murphy M., P.D. Smith, M. Wood, S. Bowden, T.J.O'Brien, K.J. Bulluss, and M.J. 
Cook. 2010. "Surgery for temporal lobe epilepsy associated with mesial temporal sclerosis in the older patient: a long term follow-up." Epilepsia 51 (6), pp. 1024-9. doi: 10.1111/j.1528-1167.2009.02430.

[16] Potter J.L., B.K. Schefft, D. W. Beebe, S.R. Howe, H.S. Yeh, and M.D. Privitera. 2009. "Presurgical neuropsychological testing predict cognitive and seizure outcomes after anterior temporal lobectomy." Epilepsy Behav 16(2):246-53. doi: 10.1016/j.yebeh.2009.07.007.

[17] Rausch R., S. Kraemer, C. J. Pietras, M. Le, B.G. Vickrey, and E.A. Passaro. 2003." Early and late cognitive changes following temporal lobe surgery for epilepsy.’Neurology 60(6):951-9.

[18] Roberson E.D., O.A. Hope, R.C. Martin, and D. Schmidt. 2011. "Geriatric epilepsy: research and clinical directions for the future." Epilepsy Behav 22 (1):103-11.

[19] Selwa L.M., S. Berent, B. Giordani, T.R. Henry, H.A. Buchtel, and D.A.Ross. 1994. "Serial Cognitive Testing in Temporal Lobe Epilepsy: Longitudinal Changes with Medical and Surgical Therapies." Epilepsia 35(4):743-9.

[20] Sirven J.I., B.L. Malamut, M.J. O'Connor, and M.R. Sperling. 2000. "Temporal lobectomy outcome in older versus younger adults." Neurology 54(11):2166-70.

[21] Srikijvilaiku T., S. Lerdlum, S. Tepmongkol, S. Shuangshoti, and C. Locharernkul. 2011. "Outcome of temporal lobectomy for hippocampal sclerosis in older patients." Seizure 20 (4):276-9. doi: 10.1016/j.seizure.2010.12.008. 